\title{
Further Specifying the Cognitive Model of Depression: Situational Expectations and Global Cognitions as Predictors of Depressive Symptoms
}

\author{
Tobias Kube $^{\mathrm{ab}}$, Philipp Herzog ${ }^{\mathrm{a}}$, Charlotte M. Michalak ${ }^{\mathrm{a}}$, Julia A. Glombiewski ${ }^{\mathrm{ab}}$, \\ Bettina K. Doering ${ }^{\text {ac }}$, Winfried Rief ${ }^{a}$ \\ [a] Department of Clinical Psychology and Psychotherapy, Philipps-University of Marburg, Marburg, Germany. \\ [b] Department of Clinical Psychology and Psychotherapy, University of Koblenz-Landau, Landau, Germany. \\ [c] Department of Psychology, Catholic University Eichstätt-Ingolstadt, Ingolstadt, Germany.
}

Clinical Psychology in Europe, 2019, Vol. 1(4), Article e33548, https://doi.org/10.32872/cpe.v1i4.33548

Received: 2019-01-31 • Accepted: 2019-04-16 • Published (VoR): 2019-12-17

Handling Editor: Cornelia Weise, Philipps-University of Marburg, Marburg, Germany

Corresponding Author: Tobias Kube, Department of Clinical Psychology and Psychotherapy, Philipps-University of Marburg, Gutenbergstraße 18, D-35032 Marburg, Germany. E-mail: tobias.kube@uni-marburg.de

\begin{abstract}
Objectives: The cognitive model of depression assumes that depressive symptoms are influenced by dysfunctional cognitions. To further specify this model, the present study aimed to examine the influence of different types of cognitions on depressive symptoms, i.e., situational expectations and global cognitions. It was hypothesized that situational expectations predict depressive symptoms beyond global cognitions.

Design: The present study examined a clinical $(\mathrm{N}=91)$ and a healthy sample $(\mathrm{N}=80)$ using longitudinal data with a baseline assessment and a follow-up five months later. Although the study was not designed as an interventional trial, participants from the clinical study received nonmanualized cognitive-behavioral treatment after the baseline assessment.

Methods: We examined situational expectations, intermediate beliefs, dispositional optimism, and generalized expectancies for negative mood regulation as predictors of depressive symptoms. Hypotheses were tested using multiple hierarchical linear regression analyses.

Results: Results indicate that, although there were significant correlations between the cognitive factors and depressive symptoms, in both samples neither global cognitions, nor situational expectations significantly predicted depressive symptoms at the five-month follow-up.

Conclusions: The present study could, contrary to the hypotheses, not provide evidence for a significant impact of cognitive vulnerabilities on depressive symptoms, presumably due to high drop-out rates at follow-up. Limitations of the study and directions for future research are critically discussed.
\end{abstract}




\section{Keywords}

expectation, expectancy, depression, cognitive model, behavioral experiment

\section{Highlights}

- Situational and global cognitions were examined as predictors of depressive symptoms.

- In a healthy and a clinical sample, cognitive factors were correlated with depressive symptoms.

- However, in both samples depressive symptoms at follow-up were not predicted by cognitive factors.

Since Beck's early studies from the 1960s (Beck, 1963, 1964), numerous studies have provided evidence for cognitive vulnerabilities among people suffering from major depressive disorder (MDD) (Mathews \& MacLeod, 2005; Scher, Ingram, \& Segal, 2005; Wenze, Gunthert, \& Forand, 2010). In particular, it has been assumed that people suffering from MDD have dysfunctional cognitions such as negative automatic thoughts, intermediate beliefs and dysfunctional core beliefs. These cognitions are supposed to influence the development and maintenance of depressive symptoms (Beck, Rush, Shaw, \& Emery, 1979). This cognitive model of depression has significantly influenced research on depression for decades and has promoted the development of cognitive-behavioral treatment. Recently, however, it has been argued that the concept of "cognition" in the traditional cognitive model might be too broad and could benefit from further specification (Rief \& Joormann, 2019). In fact, the precise influence of different types of cognitions on depressive symptoms has rarely been studied directly up to now. Therefore, the present study aimed to examine the influence of different types of cognitions that differ in their generalizability vs. specificity and the extent to which they relate to future events or experiences.

On a temporal level, cognitions can be related either to the past, the present or the future. The subgroup of cognitions that relates to future events or experiences is referred to as expectations (Kirsch, 1985; Olson, Roese, \& Zanna, 1996). More specifically, expectations may relate both to the probability of occurrence of a particular event or experience and to the consequences thereof; this can be conscious or unconscious (Laferton, Kube, Salzmann, Auer, \& Shedden Mora, 2017). While human beings are quite trained in coping with momentary unpleasant feelings, such as pain or sadness, this dramatically changes if people expect these unpleasant conditions to last forever, or to be repeated frequently in the future (Rief \& Joormann, 2019). Therefore, Rief and Joormann have argued that expectations regarding the stability of future experiences may have considerable impact on human well-being. In line with this notion, several studies have shown that negative future expectations influence the development of depressive symptoms (Horwitz, Berona, 
Czyz, Yeguez, \& King, 2017; Strunk, Lopez, \& DeRubeis, 2006; Vilhauer et al., 2012). This is consistent with Beck's 'cognitive triad' (Beck et al., 1979). The impact of negative expectations on future well-being is also supported by research on 'affective forecasting' (Wilson \& Gilbert, 2003). According to this literature, dysphoric people tend to be biased in predicting future emotional states towards the overestimation of negative emotional reactions to future events (Hoerger, Quirk, Chapman, \& Duberstein, 2012; Marroquín \& Nolen-Hoeksema, 2015).

According to Laferton et al. (2017), expectations can vary in their degree of specificity vs. generalizability. Situation-specific expectations (also referred to as 'situational expectations') such as, "When I ask someone for help, I will be rejected”, represent predictions of specific events or experiences in a particular situation. In contrast, generalized or global expectations can apply to various areas of life (e.g., "I hardly ever expect things to go my way"). Relatedly, due to their "if-then" structure, situational expectations might be more easily amenable to an empirical test of their validity compared to global expectations, e.g., through behavioral experiments. With reference to the cognitive model of depression (Beck et al., 1979), it has been hypothesized that situational expectations may constitute an important link between global beliefs, such as intermediate beliefs and dispositional optimism, and depressive symptoms. This hypothesis could recently be confirmed: the effects of both intermediate beliefs and dispositional optimism on depressive symptoms were mediated via situational expectations (Kube et al., 2018a, 2018b). To add to this line of research, the present study used longitudinal data of both healthy and depressed people to compare the predictive values of situational vs. more global cognitions in the context of depressive symptoms.

In the current work, three constructs were used as indicators for generalized cognitions. These three constructs were chosen because they have often been studied in depression research, and because there are well validated measurement tools to assess them. First, we considered dispositional optimism, arguably the most prominent concept of generalized expectations (Laferton et al., 2017). Dispositional optimism has been defined as 'the tendency to believe that one will generally experience good vs. bad outcomes in life' (Scheier \& Carver, 1985). Previous research has consistently linked optimism to depression (Korn, Sharot, Walter, Heekeren, \& Dolan, 2014; Strunk et al., 2006; Thimm, Holte, Brennen, \& Wang, 2013). Dispositional optimism can be assessed with the Life Orientation Test, the most recent form of which was presented by Scheier, Carver, and Bridges (1994). Second, another construct reflecting rather generalized expectations has been introduced by Catanzaro and Mearns (1990): they focused on generalized expectancies for negative mood regulation, and defined this construct as 'the generalized expectancy that some behavior or cognition will alleviate a negative mood state'. Similar to dispositional optimism, these expectancies, assessed with the Generalized Expectancies for Negative Mood Regulation Scale (Catanzaro \& Mearns, 1990), have been found to be associated with depressive symptoms (Backenstrass et al., 2006). Third, we considered 
intermediate beliefs, a central construct of the traditional cognitive model reflecting global attitudes and assumptions regarding oneself and life in general. Intermediate beliefs can be measured using the Dysfunctional Attitudes Scale (Oliver \& Baumgart, 1985). It has been shown that intermediate beliefs predict the development of depressive symptoms (Alloy, Abramson, Whitehouse, \& Hogan, 2006; Jarrett et al., 2012), and are associated with the severity of depressive symptoms in both healthy and clinical samples (Burns \& Spangler, 2001). Importantly, although the Dysfunctional Attitudes Scale includes items that do partly measure expectations of future events or experiences, it also comprises a considerable amount of items assessing more general attitudes without a clear focus on the future. Therefore, it cannot completely be regarded as a measure of expectations.

Besides these generalized cognitions, the current study focused on situation-specific dysfunctional expectations in depression. To assess this relatively new construct, the Depressive Expectations Scale has been developed (Kube, D'Astolfo, Glombiewski, Doering, \& Rief, 2017). Using a consequent "if-then" structure, this scale assesses situational expectations for different areas of personal and interpersonal life, such as expectations concerning social rejection, social support, mood regulation, and personal performance (see Appendix 1 for some sample items). The scale has been developed from a clinical point of view, with the aim of developing a tool that could be helpful in planning psychotherapeutic interventions to evaluate the validity of patients' expectations through behavioral experiments.

\section{Aims and Hypotheses}

The primary aim of the study was to examine situational expectations and more global cognitions as predictors of depressive symptoms. Dispositional optimism, generalized expectancies for negative mood regulation, and intermediate beliefs represent dysfunctional cognitions and have therefore conceptual similarities with situational expectations. Further, since all of these constructs reflect a negative view of an individual on different areas of personal and interpersonal life, they may only slightly differ with regards to their contents. They do differ, however, in terms of their situational specificity vs. generalizability: dispositional optimism, generalized expectancies for negative mood regulation, and intermediate beliefs represent more global cognitions while situational expectations are characterized by a higher level of situational specificity. We argue that due to this clear situational focus, situational expectations may predict depressive symptoms beyond global cognitions; situational expectations reflect specific predictions of everyday events, and therefore the actual occurrence of anticipated negative events (or the nonoccurrence of anticipated positive events) may result in negative emotions such as disappointment or frustration, thus providing the breeding ground for symptoms of depression. Global cognitions, however, are often more abstract and less closely linked to everyday experiences, so that they are less often perceived as confirmed or disconfirmed and 
thus less obviously related to depression. In particular, it was hypothesized that situational expectations at baseline predict the later severity of depressive symptoms at follow-up independently from the aforementioned more global cognitions in both a healthy and a clinical sample.

\section{Method}

This study was part of a larger research project. Recently, first data from this project using a clinical sample have recently been published (Kube et al., 2018b). The present study used the same clinical sample as the previous article. While the previous article analyzed only cross-sectional data, the present study reports the longitudinal data. Additionally, the present study reports data from a healthy sample, which have not previously been published.

\section{Participants and Procedure}

\section{Healthy Sample}

Healthy individuals were recruited via mailing lists and postings at public spaces. Inclusion criteria for the healthy sample were: absence of a currently diagnosed mental disorder (self-report), age of at least 18 years, and sufficient knowledge of the German language (self-evaluation of the participants). At baseline, 80 healthy people participated in the study and completed the questionnaires online via the commercial survey platform Unipark ${ }^{\circledR}$. Five months after the first measurement, participants were contacted by the study coordinator via Email, and they were asked to complete the questionnaires from the follow-up measure. The follow-up questionnaires were completed by 47 participants (completion rate: 58.8\%). The main reason for not completing the follow-up in the healthy sample was that participants could not be contacted again due to changes in their email address (the healthy sample consisted mainly of students, and most participants used their university e-mail address, which were no longer available if they had left the university in the meantime). Participants who completed the entire study did not significantly differ from those who completed only the baseline assessment (all $p$ values $>.05$; detailed statistical data can be found in the Appendix 2).

\section{Clinical Sample}

Participants were recruited at two inpatient hospitals $(n=53$ and $n=18)$ and one outpatient clinic $(n=24)$ in Germany. The following inclusion criteria were used: current diagnosis of MDD according to ICD-10, age of at least 18 years, sufficient knowledge of the German language (self-evaluation of the participants). In the outpatient clinic, participants were diagnosed using the SCID interview by clinical psychologists who were appropriately trained in this interview (Wittchen, Zaudig, \& Fydrich, 1997). In the inpatient 
hospitals, participants were also diagnosed by trained clinical psychologists using semistructured interviews based on SCID; these semi-structured interviews were, however, short-forms of the SCID and not validated in previous studies. If patients were interested in participating in the study, they received detailed study information and gave written informed consent. Next, participants could complete the paper-pencil questionnaires on their own. Of note, in order to control for effects of later treatment (e.g. psychotherapy), participants could only participate in the study during the first two weeks after their intake at the hospital or in diagnostic phase before the beginning of the outpatient therapy, respectively. Though the study was not an interventional trial, all participants from the clinical sample received non-manualized individual cognitive-behavioral treatment after the baseline assessment.

Five months after completing the first questionnaire, patients from the inpatient clinics were sent the second questionnaire via postal service and completed it at home. Participants from the outpatient clinic received the questionnaires by their therapists or the study coordinator. Completed follow-up questionnaires were sent back to the study coordinator, which was done by 52 persons (completion rate: $54.7 \%$ ). The reasons for not completing the follow-up assessment in the clinical sample included: participants could not be contacted again; participants were not willing to complete questionnaires again; although the participants initially agreed to complete the follow-up questionnaires, they did not return the questionnaires in the end. Completers did not significantly differ from non-completers (all $p$ values > .05; detailed statistical data can be found in the Appendix 2).

Data collection lasted from May 2016 to November 2017. For 14 participants, there were difficulties in contacting them since their contact data had changed or were incorrect. Therefore, the period of five months for the follow-up measure could not be ensured for these participants, resulting in a follow-up measure six to fourteen months after the first measurement.

For both samples, the questionnaire used at the follow-up was shorter than the one used at the first measurement, and included only the measure of situational expectations and depressive symptoms in order to decrease the anticipated drop-out rate due to additional strains. To give an incentive for participation, participants had the chance to win gift vouchers for a popular bookshop. The study was approved by local ethics committee (reference number 2016-04k) and has been conducted in accordance with ethical standards as laid down in the 1964 Declaration of Helsinki and its later amendments. All participants gave written informed consent and all procedures were in accordance with the ethical guidelines of the German Psychological Society. 


\section{Measures}

\section{Situation-Specific Dysfunctional Expectations (SDEs)}

We used the Depressive Expectations Scale (DES) to assess situation-specific dysfunctional expectations. The DES is a 25-item scale that was developed by Kube et al. (2017) to measure MDD-specific expectations. The construction principle was, according to the method of domain sampling (Nunnally, 1978), to use common dysfunctional core beliefs (e.g., "I am not likable") to deduce situation-specific predictions thereof (e.g., "When I try to make new acquaintances, I will get to know kind people" (inverted)). The majority of the items are formulated in such a way that at the beginning of each sentence a certain everyday situation is presented, and the participants' task is to indicate what behavioral, emotional, or cognitive consequences they expect to occur in this situation (see some examples in Appendix 1). Specifically, participants are asked to assess on a five-point Likert scale to what extent each of the possible completions would apply to them personally. Importantly, the completions can refer to both the likelihood of occurrence of a particular event or its emotional consequences; the main purpose of item development was simply to formulate a specific prediction that could be tested in a behavioral experiment. Since depression has been linked to both lack of positive expectations (Horwitz et al., 2017) and overly negative expectations (Strunk et al., 2006), it was important for us to balance positively and negatively worded items.

Originally, 75 items had been developed, and item reduction was performed in an online survey $(N=175)$, as described in detail in Kube et al. (2017). It resulted in a 25-item version of the DES, and a factor analysis revealed four factors: expectations of social rejection, social support, personal performance, and negative mood regulation. High sum scores of the DES reflect a greater endorsement of dysfunctional expectations. In three previous studies, the DES has shown good psychometric properties (Kube et al., 2017, 2018a, 2018b). Specifically, internal consistency ranged in previous studies between $\alpha=.87$ and $\alpha=.93$; one-year retest reliability was $r=0.430$; correlations with measures of depressive symptoms were high (correlation with the PHQ 9: $r=.754$, correlation with the BDI-II: $r=.572$ and $r=.527$, respectively). In the current study, internal consistency for the clinical sample was $\alpha=.89$ at the first measurement (for the healthy sample: $\alpha=.89$ ) and $\alpha=.92$ at follow-up (for the healthy sample: $\alpha=.94$ ). Five-months retest reliability was $r=.509$ for the clinical sample, and for the healthy sample $r=.693$. After a previous study examined the factorial structure of the DES using a convenience sample (Kube et al., 2017), we performed an exploratory factor analysis to analyze the factor structure using the clinical sample from the present study. The results of this factor analysis revealed in general a similar factor structure as in the previous study, with the exception that an additional fifth factor was found, labelled 'approval by others'. The methods, results, and discussion of this factor analysis can be found in the supplementary materials. 


\section{Dispositional Optimism}

We assessed dispositional optimism with the German version of the Life Orientation Test Revised (LOT-R), originally developed by (Scheier et al., 1994) and translated into German by Glaesmer et al. (2012). The LOT-R is a 10-item self-report scale, of which four items are distractor items and excluded when computing the sum scores. The items are rated on a five-point Likert scale. High values indicate positive outcome expectations. The LOT-R has been shown to have good reliability and validity (Glaesmer et al., 2012; Reilley, Geers, Lindsay, Deronde, \& Dember, 2005; Scheier et al., 1994). For the clinical sample from the present study, internal consistency of the LOT-R was $\alpha=.80$, and for the healthy sample it was $\alpha=.73$.

\section{Generalized Expectancies for Negative Mood Regulation}

We used the Generalized Expectancies for Negative Mood Regulation (NMR) Scale (Backenstrass et al., 2006; Catanzaro \& Mearns, 1990) to examine incremental validity of the DES over this existing measure, since the NMR scale also assesses one specific aspect of MDD-specific expectations (i.e. generalized expectancies for negative mood regulation). The NMR scale includes 30 items, and is rated using a five-point Likert scale. High values reflect positive expectations. The NMR scale has been shown to be associated with depressive symptoms, and there is evidence for good reliability of this scale (Backenstrass et al., 2006). In the current study, internal consistency for the clinical sample was $\alpha=.90$ (for the healthy sample: $\alpha=.89$ ).

\section{Intermediate Beliefs}

Intermediate beliefs were assessed using a shortened version of the Dysfunctional Attitudes Scale (DAS), originally developed by Weissman and Beck (1978) and translated into German by Hautzinger, Joormann, and Keller (2005). The 26-item version of this scale is based on those items which have consistently been shown to belong to the dimensions "performance evaluation" and "approval by others" (Cane, Olinger, Gotlib, \& Kuiper, 1986; Joormann, 2004; Prenoveau et al., 2009). Previous studies have revealed good reliability and validity of the DAS (Joormann, 2004; Nelson, Stern, \& Cicchetti, 1992). Internal consistency for the clinical sample was $\alpha=.92$ (for the healthy sample: $\alpha=.88$ ).

\section{Depressive Symptoms}

We assessed depressive symptoms with the Beck Depression Inventory-II (Beck, Steer, Ball, \& Ranieri, 1996). This well-established 21-item scale assesses somatic, cognitive and affective symptoms of depression (ranging from 0 to 63) with higher scores reflecting more severe symptoms of depression. The BDI-II has shown good psychometric properties (Beck et al., 1996). 


\section{Sociodemographic Variables}

Socio-demographic variables were assessed in a self-report questionnaire including age, sex, education, and employment status.

\section{Statistical Analyses}

Data screening was conducted according to the recommendations by Tabachnick and Fidell (2014). For four participants from the clinical sample, more than $40 \%$ of all data were missing. According to Tabachnick and Fidell (2014), these participants were excluded. Univariate outliers were inspected via standardized values of measured variables and their histograms (Kline, 2005). According to Cohen, Cohen, West, and Aiken (2003) and Stevens (2002), multivariate outliers were examined via Mahalanobis distance and Cook's distance. Data from the participants who completed the follow-up questionnaire (52 participants from the clinical sample and 47 participants from the healthy sample) were used to perform a multiple linear hierarchical regression for the two samples, separately, according to the suggestions made by Tabachnick and Fidell (2014). The MCAR test (Little, 1988) yielded non-significant results in the respective samples, indicating that the values were missing completely at random. Missing values were estimated using the expectation maximization procedure according to Tabachnick and Fidell (2014). A multivariate analysis of variance (MANOVA) examined differences between the healthy and the clinical sample.

Assumptions of multiple hierarchical linear regression analysis were carefully examined. Regression analysis was performed with the BDI-II sum scores at follow-up as dependent variables. Baseline BDI-II sum scores were included as predictors in the first block. LOT-R sum scores, NMR sum scores, and DAS sum scores from the first measurement were entered as predictors in the second block. Baseline DES sum scores were entered in the third block. Importantly, although the aforementioned constructs - DES, LOT-R, NMR expectancies and DAS - represent dysfunctional cognitions, there was no multi-collinearity between the predictors, indicated by the variance inflation factor (all values $<10$ ). In the results of the regression analyses, the standardized beta coefficients $(\beta)$ are reported. Type-1 error levels were set at $5 \%$. All analyses were conducted with IBM SPSS Statistics Version 25.

\section{Results}

\section{Sample Characteristics}

\section{Healthy Sample}

The mean participant sum score in the BDI-II at baseline was $10.10(S D=9.07)$ indicating minimum levels of depression (Beck et al., 1996). At follow-up, mean sum score in the BDI-II was $8.57(S D=10.24)$, indicating the absence of clinically relevant symptoms of 
depression. BDI-II sum scores at baseline ranged from 0 to 50 , and eleven participants reported at least moderate levels of depression (BDI-II $\geq 20$ ), of which five participants completed the follow-up questionnaire. At follow-up, BDI sum scores ranged from 0 to 46 , and four participants reported a sum score $\geq 20$. As will be shown below, the results of the main analysis are strongly influenced by whether or not the five participants who reported elevated levels of depression at baseline and completed the follow-up are included in the analysis.

A paired samples $t$-test indicated that depressive symptoms in the healthy sample did not significantly change from baseline to follow-up, $t(46)=1.054, p=.297, d=0.188$. Similarly, DES sum scores did not change from baseline $(M=49.45 ; S D=12.21)$ to follow-up $(M=49.06 ; S D=15.96), t(46)=0.227, p=.821, d=0.032$. Sample characteristics regarding socio-demographic variables are presented in Table 1.

Table 1

Sociodemographic Sample Characteristics

\begin{tabular}{|c|c|c|}
\hline Variable & Clinical sample $(N=91)$ & Healthy sample $(N=80)$ \\
\hline Age in years, $M(S D)$ & $40.8(13.2)$ & $23.05(5.32)$ \\
\hline \multicolumn{3}{|l|}{ Sex, $n(\%)^{\mathrm{a}}$} \\
\hline male & $28(31.5)$ & $20(25.0)$ \\
\hline female & $61(68.5)$ & $60(75.0)$ \\
\hline \multicolumn{3}{|l|}{ Educational level, $n(\%)^{b}$} \\
\hline No educational degree & $1(1.2)$ & 0 \\
\hline Primary education & $41(47.7)$ & $3(3.8)$ \\
\hline Secondary education & $16(18.6)$ & $57(71.3)$ \\
\hline Higher education & $28(32.6)$ & $20(25.0)$ \\
\hline \multicolumn{3}{|l|}{ Employment status, $n(\%)^{\mathrm{c}}$} \\
\hline Full-time working & $15(17.2)$ & $16(20.0)$ \\
\hline Part-time working & $6(6.9)$ & $10(12.5)$ \\
\hline In training & $12(13.8)$ & $49(61.3)$ \\
\hline Unemployed & $9(10.3)$ & $5(6.3)$ \\
\hline Disabled & $14(16.1)$ & 0 \\
\hline Be off sick & $24(27.6)$ & 0 \\
\hline Pensioners & $5(5.7)$ & 0 \\
\hline Homemaker & $2(2.3)$ & 0 \\
\hline
\end{tabular}

\section{Clinical Sample}

In our sample, $36.7 \%$ of the participants were diagnosed with a major depressive episode, $55.7 \%$ with a recurrent depressive disorder, $3.8 \%$ with a bipolar disorder, and $3.8 \%$ with a 
"double depression" (dysthymia plus current depressive episode). About a quarter of all participants $(24.7 \%)$ had at least one comorbid mental disorder with anxiety disorders being most frequent (13.5\%). The mean BDI-II sum score at baseline was $28.7(S D=9.18)$ indicating severe levels of depression (Beck et al., 1996). At the follow-up, the mean BDIII score was $19.25(S D=11.02)$ indicating moderate levels of depression. A paired samples $t$-test indicated that depressive symptoms significantly decreased from baseline to followup, $t(50)=5.205, p<.001, d=0.922$, reflecting a large effect according to Cohen (1988). SDEs also significantly changed from baseline $(M=68.71 ; S D=13.25)$ to follow-up $(M=$ 61.40; $S D=15.42), t(50)=3.583, p=.001, d=0.551$, reflecting a medium effect according to Cohen (1988). All sample characteristics regarding sociodemographic variables can be found in Table 1.

\section{Differences Between Samples}

A MANOVA indicated significant differences between the two samples (clinical vs. healthy) at baseline, $F(5,165)=73.315, p<.001, \eta_{\mathrm{p}}^{2}=.690$. Participants from the healthy sample had significantly fewer depressive symptoms, $F(1,169)=175.818, p<.001$, $\eta_{\mathrm{p}}^{2}=.510$, less pronounced situation-specific dysfunctional expectations, $F(1,169)=$ $68.775, p<.001, \eta_{\mathrm{p}}^{2}=.289$, and less pronounced generalized expectancies for negative mood regulation, $F(1,169)=51.518, p<.001, \eta_{\mathrm{p}}^{2}=.234$. They were also more optimistic, $F(1,169)=93.246, p<.001, \eta_{\mathrm{p}}^{2}=.356$, and significantly younger than those from the clinical sample, $F(1,113)=124.846, p<.001 ; \eta_{\mathrm{p}}^{2}=.425$. Frequency analyses revealed that participants from the two samples did not differ on sex distribution, $\chi^{2}=.865, p=.352$. However, healthy participants had significantly higher educational degrees, $\chi^{2}=59.371$, $p<.001$, and had, unlike the clinical sample, predominantly a student status, $\chi^{2}=69.446$, $p<.001$.

\section{Main Analysis: Prediction of Depressive Symptoms}

Using data from both samples, correlational analyses revealed significant inter-correlations of the scales used in this study, which can be found in Table 2.

Table 2

Correlational Analyses From the Healthy and Clinical Sample at Baseline

\begin{tabular}{lccccc}
\hline Sample / Variable & BDI & DES & NMR & LOT-R & DAS \\
\hline $\begin{array}{l}\text { Healthy sample } \\
\text { BDI }\end{array}$ & - & $.608^{* *}$ & $-.550^{* *}$ & $-.486^{* *}$ & $.289^{*}$ \\
DES & - & - & $-.745^{* *}$ & $-.690^{* *}$ & $.512^{* *}$ \\
NMR & - & - & - & $.685^{* *}$ & $-.490^{* *}$ \\
LOT-R & - & - & - & - & $-.371^{*}$ \\
DAS & - & - & - & - & -
\end{tabular}




\begin{tabular}{lccccc}
\hline Sample / Variable & BDI & DES & NMR & LOT-R & DAS \\
\hline Clinical sample & & & & & \\
$\quad$ BDI & - & $.641^{* *}$ & $-.520^{* *}$ & $-.522^{* *}$ & $.534^{* *}$ \\
DES & - & - & $-.672^{* *}$ & $-.561^{* *}$ & $.634^{* *}$ \\
NMR & - & - & - & $.497^{* *}$ & $-.468^{* *}$ \\
LOT-R & - & - & - & - & $-.535^{* *}$ \\
DAS & - & - & - & - & - \\
\hline
\end{tabular}

Note. BDI = Beck's Depression Inventory II; DES = Depressive Expectations Scale; NMR = Generalized Expectancies for Negative Mood Regulation Scale; LOT-R = Life Orientation Test Revised; DAS = Dysfunctional Attitudes Scale.

${ }^{*} p<.05 .{ }^{* *} p<.001$.

\section{Healthy Sample}

For the BDI-II sum scores from the follow-up as dependent variable, the baseline levels of depression explained $10.9 \%$ of the variance, and had significant effects $(\beta=.331 ; p=.023)$. The second set of predictors added another $14.7 \%$ of the explained variance which did not reach significance $(p=.054)$; none of the predictors had significant effects. Including the DES sum scores as predictors in the third block added another $5.6 \%$ of the variance, which was not significant either $(p=.074)$. The results of the regression analysis for the healthy sample can be found in Table 3.

When excluding the aforementioned five participants with elevated levels of depression, the pattern of results changed considerably. Neither BDI sum scores at baseline $\left(\Delta R^{2}=.057 ; p=.127\right)$, nor the sum scores of the LOT-R, DAS, NMR $\left(\Delta R^{2}=.011 ; p=.934\right)$, nor the sum scores of the DES at baseline $\left(\Delta R^{2}=.001 ; p=.874\right)$ predicted depressive symptoms five months later. The overall explained variance was only $6.9 \%$.

\section{Clinical Sample}

Using the BDI-II sum scores from the follow-up as dependent variables, the baseline BDIII sum scores explained $14.9 \%$ of the variance, $p=.005 ; \beta=.387$. The second set of predictors added another $7.2 \%$ of the variance $(p=.240)$. In this step, none of the predictors had significant effects. When including the DES sum scores in the third block, another $6.0 \%$ of the variance could be explained $(p=.057)$. Though there was a trend indicating the importance of DES sum scores $(\beta=.420 ; p=.057)$, none of the predictors in this step had significant effects. Results of the multiple hierarchical linear regression analysis are presented in Table 3. 
Table 3

Prediction of Depressive Symptoms in the Healthy Sample $(N=47)$ and the Clinical Sample $(N=52)$

\section{Criterion: BDI-II T2}

\section{Samples / Predictors}

Block 1

BDI-II T1

Block 2

BDI-II T1

DAS T1

NMR T1

LOT-R T1

Block 3

BDI-II T1

DAS T1

NMR T1

LOT-R T1

DES T1

$R^{2} \quad R^{2} a d j$.

\section{Model Healthy Sample}

$$
.109
$$

.090

$.109^{*}$

$5.523^{*}$

$.331^{*}$

.256

.185

.147

2.766

.103

.051

$-.172$

$-.281$

.313

.229

.056

3.366
.031

.026

$-.003$

$-.089$

.455

\section{Clinical Sample}

Block 1

.149

.132

$.145^{*}$

$8.784^{*}$

BDI-II T1

$.387^{*}$

Block 2

BDI-II T1

.256

DAS T1

.182

NMR T1

.162

LOT-R T1

$-.217$

Block 3

BDI-II T1

.173

DAS T1

.019

NMR T1

.332

LOT-R T1

$-.232$

DES T1

Note. T1 = Baseline assessment; T2 = Follow-up assessment; BDI-II = Beck Depression Inventory II; DES = Depressive Expectations Scale; DAS = Dysfunctional Attitudes Scale; NMR = Generalized Expectancies for Negative Mood Regulation Scale; LOT-R = Life Orientation Test Revised. ${ }^{*} p<.05$. 


\section{Discussion}

The aim of the present study was to examine situational expectations and more global cognitive vulnerabilities (i.e., dispositional optimism, generalized expectancies for negative mood regulation and intermediate beliefs) as predictors of depressive symptoms in a longitudinal design. In doing so, we aimed to provide a contribution to a further specification of the cognitive model of depression (Beck et al., 1979) by directly comparing the predictive values of negative global cognitions, which have been well studied in depression (Horwitz et al., 2017; Strunk et al., 2006), and situational expectations, which have recently received increasing attention (Rief et al., 2015). The results of the regression analyses indicate that for the healthy sample, none of the cognitive variables, whether global or situational, had significant effects on depressive symptoms five months later. There were only non-significant trends regarding the additionally explained variance when entering global cognitions $(p=.054)$ and situational expectations $(p=.074)$ as predictors. These trends, however, completely disappeared when excluding five participants who reported elevated levels of depression at baseline, presumably due to the thus reduced variance. In the clinical sample the effects of all cognitive variables on depressive symptoms did not reach significance either. There was merely a trend indicating the predictive value of situational expectations above global cognitions $(p=.054)$. Thus, the present study failed to provide evidence for the significance of cognitive factors as predictors of depressive symptoms.

This is in contradiction with previous studies indicating the importance of both global (Czyz, Horwitz, \& King, 2016; Horwitz et al., 2017; Strunk et al., 2006) and situational expectations (Kube et al., 2018b). Further, it is inconsistent with findings from studies examining cognitions other than expectations as predictors of depressive symptoms, such as dysfunctional attitudes (Alloy et al., 2006; Burns \& Spangler, 2001; Jarrett et al., 2012). Arguably, the absence of significant findings in the present study is, at least in the clinical sample, most likely due to the low attendance rate at follow-up. Indeed, a post-hoc power analysis indicated that the power for, e.g., detecting an incrementally significant effect of situational expectations in the clinical sample was only $43 \%$. In view of the extremely low explained variance when considering the healthy sample without participants with elevated depressive symptoms, the results of the healthy sample can best be interpreted in such a way that cognitive factors seem to have no influence whatsoever on depressive symptoms as long as there is low variability in depressive symptoms.

The non-significant results of the current study could possibly be seen as an opportunity to shift the focus away from the effects of cognitions themselves on depressive symptoms to their change in the further investigation of the cognitive model of depression. An excellent overview of directions for future research in this regard has been proposed by Lorenzo-Luaces, German, and DeRubeis (2015). In line with this notion, a series of recent studies has shown that depression is related to the absence of an optimistic bias in updating beliefs about the future (Korn et al., 2014) and inflexibility in adjusting nega- 
tive interpretations after novel positive information (Everaert, Bronstein, Cannon, \& Joormann, 2018). These findings are also in line with neurophysiological studies indicating that depression is associated with difficulty in processing unexpected events ("prediction errors”) (Garrett et al., 2014; Gradin et al., 2011).

\section{Limitations}

First and foremost, the results of the regression analyses have to be interpreted with caution due to the small sample sizes at follow-up. Given that the cognitive variables failed to reach significance in predicting depressive symptoms in the clinical sample, it is particularly important to further explore the trend regarding the importance of situational expectations as a predictor in future studies. Moreover, the small sample size at follow-up could account for the null findings regarding the influence of negative global cognitions on depressive symptoms. Therefore, future studies should aim to examine the predictive values of these variables using larger samples, ideally also including more sophisticated diagnostic procedures incl. the determination of interrater-reliability. Second, it has to be noted that the two samples considerably differed on sociodemographic variables. Therefore, it is difficult to draw inferences from the comparison of the samples. Third, given the three different clinical subsamples, multilevel methods could be considered a more sophisticated approach of analysis. However, according to a simulation study on sufficient sample sizes for multilevel analyses (Maas \& Hox, 2005), the sample size of the present study would have been at high risk of leading to biased estimates of the secondlevel standard errors, which is why we refrained from it. Fourth, as the BDI-II is a selfreport questionnaire for the measurement of depressive symptom severity, it might be useful in future studies to additionally use e.g. the Hamilton Depression Rating Scale (Hamilton, 1960) as an observer-rated assessment. Fifth, for some participants from the clinical sample, the follow-up interval was longer than five months, possibly resulting in additional variability among all participants regarding the prediction of depressive symptoms. Sixth, since all participants from the clinical sample received psychotherapeutic treatment between the two assessments, the prediction of depressive symptoms was possibly influenced by the effects of later treatment. Since the present study was not designed as an interventional study, it could not unravel specific mechanisms that may have impacted change in depressive symptoms.

In sum, the current study aimed to further specify the cognitive model of depression by directly comparing the influence of global cognitions and situational expectations on depressive symptoms. In a healthy sample and a clinical sample, the present study found neither evidence of a significant influence of global cognitions nor of situational expectations on depressive symptoms at follow-up. Given the high drop-outs at follow-up, future studies should aim to investigate larger samples in order to examine the influence of different cognitions on depressive symptoms. Special attention might also be paid in future 
studies to changes in dysfunctional cognitions and their influence on symptoms, which could be analyzed using structural equation modeling.

Funding: The authors have no funding to report.

Competing Interests: Winfried Rief is Editor-in-Chief of Clinical Psychology in Europe but played no editorial role for this particular article.

Acknowledgments: The authors have no support to report.

\section{Supplementary Materials}

The methods, results, and discussion of the exploratory factor analysis for this study are provided in the Supplementary Materials (for access, see Index of Supplementary Materials below).

\section{Index of Supplementary Materials}

Kube, T., Herzog, P., Michalak, C. M., Glombiewski, J. A., Doering, B. K., \& Rief, W. (2019). Supplementary materials to "Further specifying the cognitive model of depression: Situational expectations and global cognitions as predictors of depressive symptoms". PsychOpen. https://doi.org/10.23668/psycharchives.2655

\section{References}

Alloy, L. B., Abramson, L. Y., Whitehouse, W. G., \& Hogan, M. E. (2006). Prospective incidence of first onsets and recurrences of depression in individuals at high and low cognitive risk for depression. fournal of Abnormal Psychology, 115(1), 145-156.

https://doi.org/10.1037/0021-843X.115.1.145

Backenstrass, M., Schwarz, T., Fiedler, P., Joest, K., Reck, C., Mundt, C., \& Kronmueller, K. T. (2006). Negative mood regulation expectancies, self-efficacy beliefs, and locus of control orientation: Moderators or mediators of change in the treatment of depression? Psychotherapy Research, 16(2), 250-258. https://doi.org/10.1080/10503300500485474

Beck, A. T. (1963). Thinking and depression: I. Idiosyncratic content and cognitive distortions. Archives of General Psychiatry, 9(4), 324-333. https://doi.org/10.1001/archpsyc.1963.01720160014002

Beck, A. T. (1964). Thinking and depression II: Theory and therapy. Archives of General Psychiatry, 10(6), 561-571. https://doi.org/10.1001/archpsyc.1964.01720240015003

Beck, A. T., Rush, A., Shaw, B., \& Emery, G. (1979). Cognitive therapy of depression. New York, NY, USA: Guilford Press. 
Beck, A. T., Steer, R. A., Ball, R., \& Ranieri, W. F. (1996). Comparison of Beck Depression Inventories-IA and -II in psychiatric outpatients. Journal of Personality Assessment, 67(3), 588-597. https://doi.org/10.1207/s15327752jpa6703_13

Burns, D. D., \& Spangler, D. L. (2001). Do changes in dysfunctional attitudes mediate changes in depression and anxiety in cognitive behavioral therapy? Behavior Therapy, 32(2), 337-369. https://doi.org/10.1016/S0005-7894(01)80008-3

Cane, D. B., Olinger, L. J., Gotlib, I. H., \& Kuiper, N. A. (1986). Factor structure of the Dysfunctional Attitudes Scale in a student population. Fournal of Clinical Psychology, 42(2), 307-309. https://doi.org/10.1002/1097-4679(198603)42:2<307::AID-JCLP2270420213>3.0.CO;2-J

Catanzaro, S. J., \& Mearns, J. (1990). Measuring generalized expectancies for negative mood regulation: Initial scale development and implications. Fournal of Personality Assessment, 54(3-4), 546-563. https://doi.org/10.1080/00223891.1990.9674019

Cohen, J. (1988). Statistical power analysis for the behavioral sciences (2nd ed.). Hillsdale, NJ, USA: Erlbaum.

Cohen, J., Cohen, P., West, S. G., \& Aiken, L. S. (2003). Applied multiple regression/ correlation analysis for the behavioral sciences (3rd ed.). Mahwah, NJ, USA: Erlbaum.

Czyz, E. K., Horwitz, A. G., \& King, C. A. (2016). Self-rated expectations of suicidal behavior predict future suicide attempts among adolescent and young adult psychiatric emergency patients. Depression and Anxiety, 33(6), 512-519. https://doi.org/10.1002/da.22514

Everaert, J., Bronstein, M. V., Cannon, T. D., \& Joormann, J. (2018). Looking through tinted glasses: Depression and social anxiety are related to both interpretation biases and inflexible negative interpretations. Clinical Psychological Science, 6(4), 517-528. https://doi.org/10.1177/2167702617747968

Garrett, N., Sharot, T., Faulkner, P., Korn, C. W., Roiser, J. P., \& Dolan, R. J. (2014). Losing the rose tinted glasses: Neural substrates of unbiased belief updating in depression. Frontiers in Human Neuroscience, 8, Article 639. https://doi.org/10.3389/fnhum.2014.00639

Glaesmer, H., Rief, W., Martin, A., Mewes, R., Brähler, E., Zenger, M., \& Hinz, A. (2012). Psychometric properties and population-based norms of the Life Orientation Test Revised (LOT-R). British fournal of Health Psychology, 17, 432-445. https://doi.org/10.1111/j.2044-8287.2011.02046.x

Gradin, V. B., Kumar, P., Waiter, G., Ahearn, T., Stickle, C., Milders, M., . . Steele, J. D. (2011). Expected value and prediction error abnormalities in depression and schizophrenia. Brain, 134(6), 1751-1764. https://doi.org/10.1093/brain/awr059

Hamilton, M. (1960). A rating scale for depression. Fournal of Neurology, Neurosurgery \& Psychiatry, 23, 56-62. https://doi.org/10.1136/jnnp.23.1.56

Hautzinger, M., Joormann, J., \& Keller, F. (2005). DAS: Skala dysfunktionaler Einstellungen. Göttingen, Germany: Hogrefe.

Hoerger, M., Quirk, S. W., Chapman, B. P., \& Duberstein, P. R. (2012). Affective forecasting and selfrated symptoms of depression, anxiety, and hypomania: Evidence for a dysphoric forecasting bias. Cognition and Emotion, 26(6), 1098-1106. https://doi.org/10.1080/02699931.2011.631985 
Horwitz, A. G., Berona, J., Czyz, E. K., Yeguez, C. E., \& King, C. A. (2017). Positive and negative expectations of hopelessness as longitudinal predictors of depression, suicidal ideation, and suicidal behavior in high-risk adolescents. Suicide and Life-Threatening Behavior, 47(2), 168-176. https://doi.org/10.1111/sltb.12273

Jarrett, R. B., Minhajuddin, A., Borman, P. D., Dunlap, L., Segal, Z. V., Kidner, C. L., . . Thase, M. E. (2012). Cognitive reactivity, dysfunctional attitudes, and depressive relapse and recurrence in cognitive therapy responders. Behaviour Research and Therapy, 50(5), 280-286.

https://doi.org/10.1016/j.brat.2012.01.008

Joormann, J. (2004). The factor structure of the Dysfunctional Attitude Scale (DAS) in a non-clinical sample. Diagnostica, 50(3), 115-123. https://doi.org/10.1026/0012-1924.50.3.115

Kirsch, I. (1985). Response expectancy as a determinant of experience and behavior. American Psychologist, 40(11), 1189-1202. https://doi.org/10.1037/0003-066X.40.11.1189

Kline, R. B. (2005). Principles and practice of structural equation modeling (2nd ed.). New York, NY, USA: Guilford.

Korn, C. W., Sharot, T., Walter, H., Heekeren, H. R., \& Dolan, R. J. (2014). Depression is related to an absence of optimistically biased belief updating about future life events. Psychological Medicine, 44(3), 579-592. https://doi.org/10.1017/S0033291713001074

Kube, T., D'Astolfo, L., Glombiewski, J. A., Doering, B. K., \& Rief, W. (2017). Focusing on situationspecific expectations in major depression as basis for behavioural experiments - Development of the Depressive Expectations Scale. Psychology and Psychotherapy: Theory, Research and Practice, 90(3), 336-352. https://doi.org/10.1111/papt.12114

Kube, T., Glombiewski, J. A., \& Rief, W. (2018a). Situational expectations mediate the effect of global beliefs on depressive symptoms - A 1-year prospective study using a student sample. Current Opinion in Psychiatry, 31(5), 409-416. https://doi.org/10.1097/YCO.0000000000000443

Kube, T., Siebers, V. H. A., Herzog, P., Glombiewski, J. A., Doering, B. K., \& Rief, W. (2018b). Integrating situation-specific dysfunctional expectations and dispositional optimism into the cognitive model of depression - A path-analytic approach. fournal of Affective Disorders, 229, 199-205. https://doi.org/10.1016/j.jad.2017.12.082

Laferton, J. A. C., Kube, T., Salzmann, S., Auer, C. J., \& Shedden Mora, M. (2017). Patients' expectations regarding medical treatment: A critical review of concepts and their assessment. Frontiers in Psychology, 8, Article 233. https://doi.org/10.3389/fpsyg.2017.00233

Little, R. J. (1988). A test of missing completely at random for multivariate data with missing values. Fournal of the American Statistical Association, 83(404), 1198-1202. https://doi.org/10.1080/01621459.1988.10478722

Lorenzo-Luaces, L., German, R. E., \& DeRubeis, R. J. (2015). It's complicated: The relation between cognitive change procedures, cognitive change, and symptom change in cognitive therapy for depression. Clinical Psychology Review, 41, 3-15. https://doi.org/10.1016/j.cpr.2014.12.003

Maas, C. J., \& Hox, J. J. (2005). Sufficient sample sizes for multilevel modeling. Methodology, 1(3), 86-92. https://doi.org/10.1027/1614-2241.1.3.86 
Marroquín, B., \& Nolen-Hoeksema, S. (2015). Event prediction and affective forecasting in depressive cognition: Using emotion as information about the future. Fournal of Social and Clinical Psychology, 34(2), 117-134. https://doi.org/10.1521/jscp.2015.34.2.117

Mathews, A., \& MacLeod, C. (2005). Cognitive vulnerability to emotional disorders. Annual Review of Clinical Psychology, 1(1), 167-195. https://doi.org/10.1146/annurev.clinpsy.1.102803.143916

Nelson, L. D., Stern, S. L., \& Cicchetti, D. V. (1992). The Dysfunctional Attitudes Scale - How well can it measure depressive thinking? Journal of Psychopathology and Behavioral Assessment, 14(3), 217-223. https://doi.org/10.1007/BF00962629

Nunnally, J. C. (1978). Psychometric theory. New York, NY, USA: McGraw-Hill.

Oliver, J., \& Baumgart, E. P. (1985). The Dysfunctional Attitude Scale: Psychometric properties and relation to depression in an unselected adult population. Cognitive Therapy and Research, 9(2), 161-167. https://doi.org/10.1007/BF01204847

Olson, J. M., Roese, N. J., \& Zanna, M. P. (1996). Expectancies. In E. T. Higgins \& A. W. Kruglanski (Eds.), Social psychology: Handbook of basic principles (pp. 211-238). New York, NY, USA: Guilford Press.

Prenoveau, J. M., Zinbarg, R. E., Craske, M. G., Mineka, S., Griffith, J. W., \& Rose, R. D. (2009). Evaluating the invariance and validity of the structure of dysfunctional attitudes in an adolescent population. Assessment, 16(3), 258-273. https://doi.org/10.1177/1073191108324519

Reilley, S. P., Geers, A. L., Lindsay, D. L., Deronde, L., \& Dember, W. N. (2005). Convergence and predictive validity in measures of optimism and pessimism sequential studies. Current Psychology, 24(1), 43-59. https://doi.org/10.1007/s12144-005-1003-z

Rief, W., Glombiewski, J. A., Gollwitzer, M., Schubo, A., Schwarting, R., \& Thorwart, A. (2015). Expectancies as core features of mental disorders. Current Opinion in Psychiatry, 28(5), 378-385. https://doi.org/10.1097/YCO.0000000000000184

Rief, W., \& Joormann, J. (2019). Revisiting the cognitive model of depression: The role of expectations. Clinical Psychology in Europe, 1(1), Article e32605. https://doi.org/10.32872/cpe.v1i1.32605

Scheier, M. F., \& Carver, C. S. (1985). Optimism, coping, and health - Assessment and implications of generalized outcome expectancies. Health Psychology, 4(3), 219-247.

https://doi.org/10.1037/0278-6133.4.3.219

Scheier, M. F., Carver, C. S., \& Bridges, M. W. (1994). Distinguishing optimism from neuroticism (and trait anxiety, self-mastery, and self-esteem) - A reevaluation of the Life Orientation Test. Journal of Personality and Social Psychology, 67(6), 1063-1078. https://doi.org/10.1037/0022-3514.67.6.1063

Scher, C. D., Ingram, R. E., \& Segal, Z. V. (2005). Cognitive reactivity and vulnerability: Empirical evaluation of construct activation and cognitive diatheses in unipolar depression. Clinical Psychology Review, 25(4), 487-510. https://doi.org/10.1016/j.cpr.2005.01.005

Stevens, J. (2002). Applied multivariate statistics for the social sciences (2nd ed.). Hillsdale, NJ, USA: Erlbaum. 
Strunk, D. R., Lopez, H., \& DeRubeis, R. J. (2006). Depressive symptoms are associated with unrealistic negative predictions of future life events. Behaviour Research and Therapy, 44(6), 861-882. https://doi.org/10.1016/j.brat.2005.07.001

Tabachnick, B. G., \& Fidell, L. S. (2014). Using multivariate statistics (6th ed.). Harlow, United Kingdom: Pearson.

Thimm, J. C., Holte, A., Brennen, T., \& Wang, C. (2013). Hope and expectancies for future events in depression. Frontiers in Psychology, 4, Article 470. https://doi.org/10.3389/fpsyg.2013.00470

Vilhauer, J. S., Young, S., Kealoha, C., Borrmann, J., IsHak, W. W., Rapaport, M. H., . . Mirocha, J. (2012). Treating major depression by creating positive expectations for the future: A pilot study for the effectiveness of Future-Directed Therapy (FDT) on symptom severity and quality of life. CNS Neuroscience \& Therapeutics, 18(2), 102-109. https://doi.org/10.1111/j.1755-5949.2011.00235.x

Weissman, A. N., \& Beck, A. T. (1978). Development and validation of the Dysfunctional Attitude Scale: A preliminary investigation. Paper presented at the Annual Meeting of American Educational Research Association, Toronto, Canada.

Wenze, S. J., Gunthert, K. C., \& Forand, N. R. (2010). Cognitive reactivity in everyday life as a prospective predictor of depressive symptoms. Cognitive Therapy and Research, 34(6), 554-562. https://doi.org/10.1007/s10608-010-9299-x

Wilson, T. D., \& Gilbert, D. T. (2003). Affective forecasting. Advances in Experimental Social Psychology, 35(35), 345-411. https://doi.org/10.1016/S0065-2601(03)01006-2

Wittchen, H., Zaudig, M., \& Fydrich, T. (1997). Structured clinical interview for DSM-IV. Göttingen, Germany: Hogrefe.

\section{Appendices}

\section{Appendix 1: Sample Items of the DES}

- Expectations regarding social rejection: e.g., "When I ask someone for help, I will be rejected"

- Expectations regarding social support: e.g., "When I talk to someone about my problems, I will feel better afterwards" (inverted)

- Expectations regarding mood regulation: e.g., "When I'm feeling guilty, I will feel better when I lie down in my bed"

- Expectations regarding personal performance: e.g., "When I have to get an important task done, I will fail at it"

\section{Appendix 2: Completer Analyses}

Participants from the healthy sample who completed the entire study did not significantly differ from those healthy people who completed only the baseline assessment in baseline depressive symptoms, $F(1,78)=0.066, p=.798 ; \eta_{\mathrm{p}}^{2}=.001$; situational expectations, $F(1,78)=2.811, p=.098$; $\eta_{\mathrm{p}}^{2}=.035$; dispositional optimism, $F(1,78)=3.164, p=.079 ; \eta_{\mathrm{p}}^{2}=.039$; intermediate beliefs, $F(1,78)=$ $0.683, p=.411 ; \eta_{\mathrm{p}}^{2}=.009$; expectancies for negative $\operatorname{mood}$ regulation, $F(1,78)=0.476, p=.492$; 
$\eta_{\mathrm{p}}^{2}=.006$; age, $F(1,78)=0.105, p=.746 ; \eta_{\mathrm{p}}^{2}=.001$; sex, $\chi^{2}(1)=2.080, p=.149$; education, $\chi^{2}(2)=$ $0.560, p=.756$; or employment status, $\chi^{2}(3)=2.674, p=.445$.

Similarly, in the clinical sample completers did not significantly differ from non-completers in baseline depressive symptoms, $F(1,89)=2.384, p=.126 ; \eta_{\mathrm{p}}^{2}=.026$; situational expectations, $F(1$, $89)=0.126, p=.723 ; \eta_{\mathrm{p}}^{2}=.001$; dispositional optimism, $F(1,89)=0.598, p=.442 ; \eta_{\mathrm{p}}^{2}=.007$; intermediate beliefs, $F(1,89)=0.058, p=.810 ; \eta_{\mathrm{p}}^{2}=.001$; expectancies for negative mood regulation, $F(1$, $89)=0.050, p=.823 ; \eta_{\mathrm{p}}^{2}=.001$; age, $F(1,89)=3.702, p=.058 ; \eta_{\mathrm{p}}^{2}=.040$; primary diagnosis, $\chi^{2}(3)=$ 4.095, $p=.251$; comorbid diagnosis, $\chi^{2}(7)=4.195, p=.757$; sex, $\chi^{2}(1)=1.975, p=.160$; education, $\chi^{2}(5)=6.715, p=.243$; or employment status, $\chi^{2}(7)=3.738, p=.809$.

\section{EACLIPT}

Clinical Psychology in Europe (CPE) is the official journal of the European Association of Clinical Psychology and Psychological Treatment (EACLIPT).

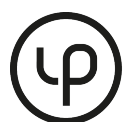

leibniz-psychology.org

PsychOpen GOLD is a publishing service by Leibniz Institute for Psychology Information (ZPID), Germany. 